\title{
Pharmacotherapeutic considerations for systemic rheumatic diseases amid the COVID-19 pandemic: more questions than answers
}

\author{
Chia Siang Kow ${ }^{1} \mathbb{D} \cdot$ Syed Shahzad Hasan ${ }^{2}$
}

Published online: 16 August 2020

C) Springer Nature Switzerland AG 2020

\begin{abstract}
Thus far, associations between the presence of systemic rheumatic disease and an increased risk of novel coronavirus disease 2019 (COVID-19) acquisition or a worse prognosis from COVID-19 have not been conclusive. It is not known for certain if there is an association between any pharmacological agent used for rheumatologic treatment, including biological and non-biological disease-modifying antirheumatic drugs (DMARDs), and an increased risk of COVID-19 acquisition or adverse outcomes from COVID-19, although these agents have been associated with an overall higher risk of infections. The pharmacological management of patients with a rheumatic disease without COVID-19 should currently follow usual treatment approaches. Individualized approaches to adjusting DMARD regimens in patients with documented COVID-19 seems prudent, with specific attention paid to the severity of the infection. Patients receiving antimalarials (hydroxychloroquine/ chloroquine) may continue treatment with these agents. Treatment with sulfasalazine, methotrexate, leflunomide, immunosuppressants and biological agents other than interluekin- 6 receptor inhibitors and JAK inhibitors should be stopped or withheld. It should be reasonable to resume DMARD treatment when patients are no longer symptomatic and at least 2 weeks after documentation of COVID-19, although the decision should be individualized, preferably based on infection severity.
\end{abstract}

\section{Introduction}

In late 2019, an outbreak of pneumonia cases was reported in Wuhan, a city in the Hubei province of China, which has later been termed novel coronavirus disease 2019 (COVID19). By 2020, COVID-19 rapidly escalated to a pandemic that spread to almost all countries in the world. A novel coronavirus, severe acute respiratory syndrome coronavirus 2 (SARS-CoV-2), was identified as the causeative pathogen of COVID-19. In this opinion paper, we discuss the pharmacotherapeutic considerations of patients with systemic rheumatic diseases during the COVID-19 pandemic based on currently available evidence (as of 25 July 2020).

Chia Siang Kow

chiasiang_93@hotmail.com

1 School of Postgraduate Studies, International Medical University, No. 126, Jln Jalil Perkasa 19, Bukit Jalil, 57000 Kuala Lumpur, Malaysia

2 School of Applied Sciences, University of Huddersfield, Huddersfield, UK

\section{Are patients with systemic rheumatic diseases at increased risk of acquiring COVID-19?}

Although otherwise healthy individuals can acquire COVID19 or develop a severe course of the disease, it is more likely among patients with underlying medical comorbidities [1, 2]. Nevertheless, thus far, there is no reported association between the presence of systemic rheumatic disease (e.g. rheumatoid arthritis, psoriatic arthritis, ankylosing spondylitis, systemic lupus erythematosus, etc.) and increased risk of COVID-19 acquisition or even a worse prognosis from COVID-19. In one prospective case series of 86 patients in New York with immune-mediated inflammatory diseases (24\% with psoriatic arthritis, $23 \%$ with rheumatoid arthritis and $10 \%$ with ankylosing spondylitis) diagnosed with or presumed to have COVID-19, the proportion of patients requiring hospitalization (14 of 86 patients; $16 \%$ ) was similar to the general population in New York $(26 \%$ at the time of reporting) [3]. In another case series from the Lombardy region of Italy, among 320 patients with chronic arthritis receiving a disease-modifying antirheumatic drug (DMARD), eight patients were diagnosed with or strongly suspected of COVID-19, but only one patient required 
hospital admission [4]. A comparison with $>33,000$ cases of COVID-19 in this region including $>1250$ patients requiring admission to intensive care units (ICUs) during this period suggests the risk of COVID-19 acquisition did not increase in patients with chronic arthritis. Similar findings were described among 859 patients surveyed by telephone who were receiving DMARDs for rheumatic diseases and sarcoidosis in Siena, Italy, where only two of these patients had been diagnosed with COVID-19 [5]. Furthermore, a nationwide study in Russia found that the prevalence of systemic rheumatic diseases among COVID-19 patients admitted to ICUs with a severe course of COVID-19 was low (10 of 902 patients; $1.1 \%$ ) and similar to that of the general Russian population [6]. Similar to their adult counterparts, paediatric patients with systemic rheumatic disease appear to be at no increased risk for the acquisition of COVID-19 [7, 8].

Despite no reported direct association, it is noteworthy that patients with rheumatic diseases are frequently of older age and have comorbidities such as chronic pulmonary disorders, chronic kidney disease, cardiovascular disease (CVD), hypertension, obesity and diabetes mellitus. These comorbidities, in turn, are risk factors for a severe course of COVID-19 [9-15]. For instance, a meta-analysis of 24 observational studies that included 111,758 patients reported a 59\% higher risk of mortality from coronary artery disease in patients with rheumatoid arthritis relative to the general population [16]. In addition, a systematic review of 28 studies concluded that patients with systemic lupus erythematosus had at least double the risk of CVD (myocardial infarction, cerebrovascular disease and peripheral vascular disease) compared with the general population [17].

\section{Does the use of DMARDs effect the clinical outcomes of COVID-19?}

It is not known definitely whether there is an association between the use of any DMARD, including biological and non-biological agents, and an increased risk of COVID-19 acquisition or adverse outcomes from COVID-19. However, pharmacological agents used to treat systemic rheumatic diseases have been associated with an overall higher risk of infections in both adult and paediatric populations [18, 19]. Findings from emerging observational studies have been mixed. To illustrate, the aforementioned prospective case series [3] that included 86 patients from New York with confirmed or presumptive COVID-19 with concurrent immunemediated inflammatory disease reported no increased odds of COVID-19 hospitalization among those who were receiving biological agents or Janus kinase (JAK) inhibitors at baseline [adjusted odds ratio (OR) 0.85 ; 95\% CI 0.71-1.02]. In contrast, patients receiving methotrexate at baseline were at significantly increased odds of being hospitalized for COVID-19 (adjusted OR 1.29; 95\% CI 1.04-1.58).

On the other hand, a single-centre case-control study [20] in the Lombardy region of Italy that included 1193 patients with psoriasis receiving either biological agents or conventional small-molecule drugs has reported otherwise. Patients treated with biological agents had significantly higher odds of developing symptomatic COVID-19 infection (OR 3.43; 95\% CI 2.25-5.73), being self-quarantined at home (OR 9.05; 95\% CI 5.61-14.61) and being hospitalized for COVID-19 (OR 3.59; 95\% CI 1.49-8.63) than the general population in the region. The odds of admission to an ICU or of death did not differ, although there had been insufficient number of patients in the study to provide adequate statistical power [20].

In a multinational case series of 600 patients with rheumatic diseases and COVID-19 included in the COVID-19 Global Rheumatology Alliance physician-reported registry, the use of a tumour necrosis factor inhibitor was associated with lower odds of hospitalization (OR 0.40; 95\% CI 0.19-0.81) [21]. There was no significant association between the use of conventional DMARDs, either alone or in combination with biologicals/JAK inhibitors, and an increased risk of hospitalization for COVID-19 [21].

\section{Should DMARD treatment regimens be adjusted?}

Based on currently available evidence, we feel that pharmacological management for patients with newly diagnosed, stable, or active systemic rheumatic disease without COVID19 should follow usual treatment approaches. There are proven benefits for initiation or continuation of any pharmacological agents for the management of systemic rheumatic diseases, including treatment with conventional DMARDs and other immunosuppressive agents (e.g. hydroxychloroquine/chloroquine, sulfasalazine, methotrexate, leflunomide, tacrolimus, ciclosporin, mycophenolate mofetil and azathioprine), as well as biological DMARDs, such as abatacept, tocilizumab and JAK inhibitors (e.g. tofacitinib, baricitinib and upadacitinib).

Without conclusive proof from clinical trials, an individualized approach to adjusting DMARD regimens in patients with documented or presumptive COVID-19 seems prudent, with specific attention paid to severity of illness. Management practices recommended by various professional societies of rheumatology can be useful guidance for clinicians managing documented or presumptive COVID-19 patients with systemic rheumatic diseases [22-26]. The decision to either continue/ discontinue the use of DMARDs should be made in close 
consultation with the experts in infectious disease and critical care involved in the management of the patient's acute illness.

Patients receiving antimalarials (hydroxychloroquine/ chloroquine) may continue these agents, although they may require electrocardiographic screening and monitoring given the potential for prolonged QT interval and possible increased risk of cardiotoxicity in the context of COVID-19. The recommendation was based upon a low risk of serious infection associated with hydroxychloroquine/chloroquine, as well as the potential benefit of preventing a flare-up from systemic rheumatic diseases. Nonetheless, concomitant use of medications that can prolong the QT interval should be reduced, if not completely be avoided, when the decision is made to continue hydroxychloroquine/chloroquine treatment.

Sulfasalazine, methotrexate, leflunomide, immunosuppressants (e.g. mycophenolate mofetil, azathioprine) and biological agents, other than interleukin (IL)-6 receptor inhibitors and JAK inhibitors, should be discontinued temporarily in patients with documented or presumptive COVID-19. The rationale for withholding sulfasalazine in the context of COVID-19 is due to concerns regarding the potential confusion of adverse effects from sulfasalazine (e.g. gastrointestinal upset, diarrhoea, hepatitis, cytopenias and pneumonitis) with the clinical manifestations of COVID-19. Theoretically, withholding sulfasalazine for up to 2 or 3 weeks has a low likelihood of resulting in significant rheumatic disease flares. Meanwhile, temporary discontinuation of methotrexate, leflunomide, immunosuppressants and biological agents (other than IL-6 receptor and JAK inhibitors) is mainly related to the growing concerns over possible impairment in the host defence mechanism against SARS-CoV-2 and the risk of secondary bacterial or opportunistic infections.

Likewise, the theoretical risk of a flare-up from rheumatic diseases is likely to be relatively low during the limited period in which DMARDs are being withheld, while the risk of secondary bacterial or opportunistic infections should not be ignored, as it is now recognized that a substantial proportion of patients with COVID-19 may develop secondary bacterial pneumonia [1]. On the other hand, IL-6 receptor inhibitors (e.g. tocilizumab and sarilumab) may be continued in selected patients, particularly those in whom cytokine storm syndrome is impending, preferably in clinical trial settings, given the potential benefit of IL-6 inhibitors in COVID-19 patients who develop an intense inflammatory state; however, conclusive evidence on their efficacy remains to be elucidated [27-33].

\section{When should DMARDs be resumed after documented COVID-19?}

There are very limited data to address the issue on the optimal time to resume DMARDs after a diagnosis of COVID19 , but we think it should be reasonable to resume when patients are no longer symptomatic and at least 2 weeks have passed since the documentation of COVID-19. More information regarding how long COVID-19 patients remain infectious is needed. Without conclusive data as yet, there is no 'one size fits all' approach regarding the time to resumption of DMARDs; therefore, the approach should be individualized, preferably based on the severity of illness.

It has been demonstrated that replication-competent (live/ viable) SARS-CoV-2 has not been recovered up to 9 days from symptom onset in patients with mild-to-moderate COVID-19. To illustrate, in a small observational study in nine COVID-19 patients [34], seroconversion was detected in all patients after 2 weeks, while viral loads progressively decreased, and the live virus was unable to be isolated 8 days after symptom onset. Similarly, in a cross-sectional study that analysed 90 SARS-CoV-2-positive samples that ranged from the day of symptom onset to 21 days post-symptom onset, positive cultures were only observed up to 8 days post-symptom onset [35]. In another study, isolation of viable SARS-CoV-2 from nasopharyngeal and oropharyngeal swabs in 31 COVID-19 patients was possible only up to 9 days after symptom onset [36].

Recovery of replication-competent SARS-CoV-2 10-20 days after symptom onset has been documented in some patients with a severe course of COVID-19 complicated by an immunocompromised state [37]. In a series of 129 COVID-19 patients with a severe or critical illness, of whom 30 were immunocompromised, viable SARS-CoV-2 was detected in a single patient up to 20 days after onset of symptoms [37]. Nevertheless, it was estimated that $95 \%$ of the samples no longer yielded a replication-competent virus 15 days following symptom onset [37].

\section{More questions than answers}

In conclusion, we have more questions than answers in the management of rheumatology patients in the context of COVID-19. Future studies should aim to answer the following questions raised in our discussion:

- Does the presence of systemic rheumatic disease increase the risk of COVID-19 acquisition or a worse prognosis from COVID-19?

- Does the baseline use of rheumatologic treatment, including biological and non-biological agents, increase the risk of COVID-19 acquisition or adverse outcomes?

- Do the benefits of initiation or continuation of pharmacological agents for the management of (newly) diagnosed systemic rheumatic diseases outweigh the risks of COVID-19 acquisition?

- Should pharmacological regimens for the management of systemic rheumatic diseases be adjusted (continued/ 
discontinued) in patients with documented or presumptive COVID-19?

- When is the optimal time for the resumption of antirheumatic drugs after COVID-19?

\section{Take-home messages}

- Be aware that the presence of rheumatic disease alone is not yet associated with an increased risk for acquiring COVID-19.

- Individualize adjustments to rheumatic medications in patients with COVID-19, taking infection severity into consideration.

- In the absence of COVID-19, generally follow the usual treatment approaches and recommendations for managing patients with newly diagnosed or stable rheumatic disease.

Author contributions CSK and SSH contributed equally to the writing of this manuscript.

\section{Declarations}

Conflict of interest The authors have no potential conflict of interest to declare.

Funding No funding was received.

Ethics approval, Consent to participate, Consent for publication, Availability of data and materials and Code availability None applicable.

\section{References}

1. Zhou F, Yu T, Du R, et al. Clinical course and risk factors for mortality of adult inpatients with COVID-19 in Wuhan, China: a retrospective cohort study. Lancet. 2020;395(10229):1054-62.

2. Wu Z, McGoogan JM. Characteristics of and important lessons from the coronavirus disease 2019 (COVID-19) outbreak in China: summary of a report of 72314 cases from the Chinese Center for Disease Control and Prevention. JAMA. 2020. https:// doi.org/10.1001/jama.2020.2648.

3. Haberman R, Axelrad J, Chen A, et al. Covid-19 in immunemediated inflammatory disease: case series from New York. $\mathrm{N}$ Engl J Med. 2020;383(1):85-8.

4. Mikuls TR. Co-morbidity in rheumatoid arthritis. Best Pract Res Clin Rheumatol. 2003;17(5):729-52.

5. Conticini E, Bargagli E, Bardelli M, et al. COVID-19 pneumonia in a large cohort of patients treated with biological and targeted synthetic antirheumatic drugs. Ann Rheum Dis. 2020. https://doi. org/10.1136/annrheumdis-2020-217681.

6. Moiseev S, Avdeev S, Brovko M, et al. Rheumatic diseases in intensive care unit patients with COVID-19. Ann Rheum Dis. 2020. https://doi.org/10.1136/annrheumdis-2020-217676.

7. Costi S, Caporali R, Cimaz R. Dealing with COVID-19 in a pediatric rheumatology unit in Italy. Paediatr Drugs. 2020;22(3):263-4.
8. Mukusheva Z, Assylbekova M, Poddighe D. Management of pediatric rheumatic patients in Kazakhstan during the coronavirus disease 2019 (COVID-19) pandemic. Rheumatol Int. 2020;40(8):1351-2.

9. Nurmohamed MT, Heslinga M, Kitas GD. Cardiovascular comorbidity in rheumatic diseases. Nat Rev Rheumatol. 2015;11(12):693-704.

10. Bichile T, Petri M. Prevention and management of co-morbidities in SLE. Presse Med. 2014;43(6 Pt 2):e187-95.

11. Perez-Chada LM, Merola JF. Comorbidities associated with psoriatic arthritis: review and update. Clin Immunol. 2020;214:108397.

12. Burner TW, Rosenthal AK. Diabetes and rheumatic diseases. Curr Opin Rheumatol. 2009;21(1):50-4.

13. Queiro R, Lorenzo A, Tejón P, et al. Obesity in psoriatic arthritis: comparative prevalence and associated factors. Medicine (Baltimore). 2019;98(28):e16400.

14. Panoulas VF, Metsios GS, Pace AV, et al. Hypertension in rheumatoid arthritis. Rheumatology (Oxford). 2008;47(9):1286-98.

15. Aviña-Zubieta JA, Choi HK, Sadatsafavi M, et al. Risk of cardiovascular mortality in patients with rheumatoid arthritis: a meta-analysis of observational studies. Arthritis Rheum. 2008;59(12):1690-7.

16. Schoenfeld SR, Kasturi S, Costenbader KH. The epidemiology of atherosclerotic cardiovascular disease among patients with SLE: a systematic review. Semin Arthritis Rheum. 2013;43(1):77-95.

17. Atzeni F, Masala IF, di Franco M, et al. Infections in rheumatoid arthritis. Curr Opin Rheumatol. 2017;29(4):323-30.

18. Sepriano A, Kerschbaumer A, Smolen JS, et al. Safety of synthetic and biological DMARDs: a systematic literature review informing the 2019 update of the EULAR recommendations for the management of rheumatoid arthritis. Ann Rheum Dis. 2020;79(6):760-70.

19. Poddighe D, Romano M, Gattinara M, et al. Biologics for the treatment of juvenile idiopathic arthritis. Curr Med Chem. 2018;25(42):5860-93.

20. Damiani G, Pacifico A, Bragazzi NL, et al. Biologics increase the risk of SARS-CoV-2 infection and hospitalization, but not ICU admission and death: real-life data from a large cohort during red-zone declaration. Dermatol Ther 2020; 13475.

21. Gianfrancesco M, Hyrich KL, Al-Adely S, et al. Characteristics associated with hospitalisation for COVID-19 in people with rheumatic disease: data from the COVID-19 Global Rheumatology Alliance physician-reported registry. Ann Rheum Dis. 2020;79(7):859-66.

22. Mikuls TR, Johnson SR, Fraenkel L, et al. American College of Rheumatology guidance for the management of adult patients with rheumatic disease during the COVID-19 pandemic: version 1 . Arthritis Rheumatol. 2020. https://doi.org/10.1002/art.41301.

23. COVID-19 rapid guideline: rheumatological autoimmune, inflammatory and metabolic bone disorders. NICE guideline [NG167] Published date: 03 April 2020 Last updated: 21 May 2020. https ://www.nice.org.uk/guidance/ng167. Accessed 23 May 2020.

24. Schulze-Koops H, Specker C, Iking-Konert C, et al. Preliminary recommendations of the German Society of Rheumatology (DGRh $\mathrm{eV}$ ) for the management of patients with inflammatory rheumatic diseases during the SARS-CoV-2/COVID-19 pandemic. Ann Rheum Dis. 2020;79(6):840-2.

25. Matucci-Cerinic M, Bruni C, Allanore Y, et al. Systemic sclerosis and the COVID-19 pandemic: World Scleroderma Foundation preliminary advice for patient management. Ann Rheum Dis. 2020;79(6):724-6.

26. Landewé RB, Machado PM, Kroon F, et al. EULAR provisional recommendations for the management of rheumatic and musculoskeletal diseases in the context of SARS-CoV-2. Ann Rheum Dis. 2020;79(7):851-8. 
27. Mehta P, McAuley DF, Brown M, et al. COVID-19: consider cytokine storm syndromes and immunosuppression. Lancet. 2020;395(10229):1033-4.

28. Luo P, Liu Y, Qiu L, et al. Tocilizumab treatment in COVID-19: a single center experience. J Med Virol. 2020;92(7):814-8.

29. Michot JM, Albiges L, Chaput N, et al. Tocilizumab, an anti-IL6 receptor antibody, to treat COVID-19-related respiratory failure: a case report. Ann Oncol. 2020;31(7):961-4.

30. Zhang X, Song K, Tong F, et al. First case of COVID-19 in a patient with multiple myeloma successfully treated with tocilizumab. Blood Adv. 2020;4(7):1307-10.

31. Sciascia $\mathrm{S}, \mathrm{Aprà} \mathrm{F}, \mathrm{Baffa} \mathrm{A}$, et al. Pilot prospective open, singlearm multicentre study on off-label use of tocilizumab in patients with severe COVID-19. Clin Exp Rheumatol. 2020;38(3):529-32.

32. Klopfenstein T, Zayet S, Lohse A, et al. Tocilizumab therapy reduced intensive care unit admissions and/or mortality in COVID-19 patients. Med Mal Infect. 2020;50(5):397-400.
33. Xu X, Han M, Li T, et al. Effective treatment of severe COVID19 patients with tocilizumab. Proc Natl Acad Sci USA. 2020;117(20):10970-5.

34. Wölfel R, Corman VM, Guggemos W, et al. Virological assessment of hospitalized patients with COVID-2019. Nature. 2020;581(7809):465-9.

35. Bullard J, Dust K, Funk D, et al. Predicting infectious SARSCoV-2 from diagnostic samples. Clin Infect Dis. 2020; ciaa638.

36. Arons MM, Hatfield KM, Reddy SC, et al. Presymptomatic SARS-CoV-2 infections and transmission in a skilled nursing facility. N Engl J Med. 2020;382(22):2081-90.

37. van Kampen J, van de Vijver D, Fraaij P, et al. Shedding of infectious virus in hospitalized patients with coronavirus disease-2019 (COVID-19): duration and key determinants. medRxiv. 2020. https://doi.org/10.1101/2020.06.08.20125310. 